EPJ Web of Conferences 52, 09002 (2013)

DOI: $10.1051 /$ epjconf/20135209002

(C) Owned by the authors, published by EDP Sciences, 2013

\title{
Theory Predictions for Inclusive atmospheric Neutrino flux
}

\author{
Morihiro Honda ${ }^{1, a}$ \\ ${ }^{1}$ Institute for Cosmic Ray Research, University of Tokyo, Kashiwa, Chiba, 277-85823, Japan
}

\begin{abstract}
We describe the history of theory prediction of inclusive atmospheric neutrino flux shortly, then the 3-dimensional calculation of atmospheric neutrino flux in some detail. With the calculated atmospheric neutrino flux for INO and South Pole, we discuss on the relation of atmospheric neutrino flux and geomagnetic field. We find the full 3-dimensional scheme calculation is necessary for the theory prediction of the atmospheric neutrino flux.
\end{abstract}

\section{Short history as the introduction}

The atmospheric neutrino flux is calculated in the 1dimensional scheme for long time[1, 2]. It is believed that the 1-dimensional scheme calculation is good enough because of the nature of the hadronic interaction and the small muon bending angle in the geomagnetic field $(-5$ degree) before the decay. Also the 3-dimensional scheme calculation is very inefficient computation compared to the 1dimensional scheme calculation. We had considered it is impossible to complete within a reasonable computation time.

These situation continued until Fluka group [3] reported the calculation in a 3-dimensional scheme, limiting the effect of geomagnetic field only to the rigidity cutoff. In this scheme of the calculation, they could reduce the computation time largely, but find a large horizontal enhancement of neutrino flux, which is not seen in the 1dimensional scheme calculation.

The importance of the geomagnetic field in the atmosphere is discussed by Lipari [4] qualitatively, and a 3-dimensional scheme calculation with the geomagnetic field in the atmosphere was carried out by Barr et al. [5], supporting the discussion of Lipari quantitatively. However, they introduced many acceleration technique in their calculation. It is not a full 3-dimensional calculation yet.

A calculation a little more close to the full 3dimensional scheme was carried out by Honda et al. [6], with fast interaction code and virtual detector correction. We illustrate this work and the following developments in this paper.

\section{Calculation Scheme}

For the calculation of atmospheric neutrino flux, we need the primary cosmic ray spectra model, the atmosphere model, and the geomagnetic field model, other than the

\footnotetext{
ae-mail: mhonda@icrr.u-tokyo.ac.jp
}

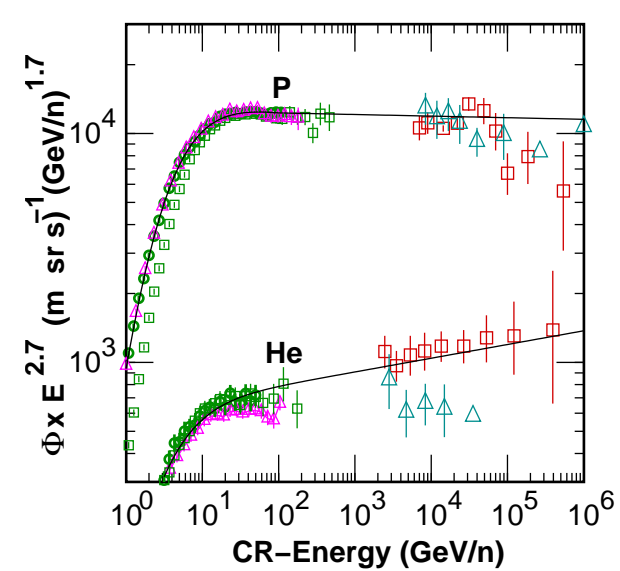

Figure 1. Primary cosmic ray data and the spectra model. Small triangles show the AMS-01 [7] data, small circles BESS [8], small squares BESS-TeV [9], Large triangles JACEE [12], and large squares RUNJOB [13].

hadronic interaction model. We used the primary flux model based on AMS [7] and BESS [8, 9] data shown in Fig. 1. US-standard76 [10] is used for the atmosphere model, and IGRF2000 [11] for the geomagnetic model in Ref [6]. Those atmosphere and geomagnetic models are taken over by NRLMSISE-00 [10] and IGRF2010 [11] respectively, in the more recent work [14]. We show the horizontal component of the geomagnetic field in Fig. 2 with the 2 experimental sites where we calculate the atmospheric neutrino flux in this paper (INO [15] and South Pole [16]).

For the 3-dimensional calculation, we assumed the surface of the earth is a sphere with radius of $R_{e}=63781.80$ $\mathrm{km}$. In addition to the surface of the earth we assumed three more spheres, the injection sphere, the simulation sphere, and the escape sphere. We have taken the radius of the injection sphere as $R_{\mathrm{i} n j}=R_{e}+100 \mathrm{~km}$, and the radius of simulation sphere as $R_{\mathrm{esc}}=R_{\mathrm{sim}}=3 \times R_{e}$, and that of escape sphere as $R_{\mathrm{e} s c}=R_{\mathrm{s} i m}=10 \times R_{e}=63781.80 \mathrm{~km}$ 


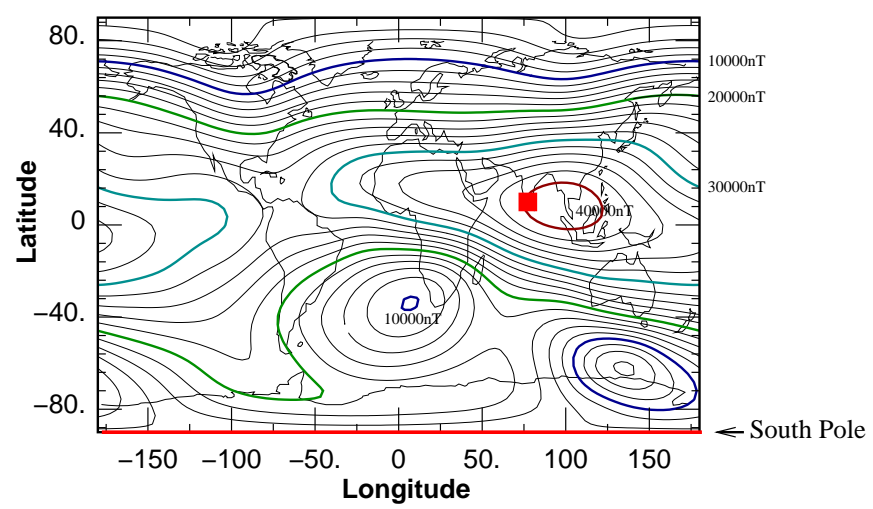

Figure 2. Magnitude of the horizontal component of geomagnetic field in IGRF2010 model [11]. Square stands for the position of India-based Neutrino Observation (INO) site [15], and the bottom bar for the South Pole [16].

in Ref. [6]. The radius of simulation sphere is take as the same as that of escape sphere $\left(R_{\mathrm{e} s c}=R_{\mathrm{sim}}=10 \times R_{e}\right)$ after Ref. [17].

Cosmic rays are sampled on the injection sphere uniformly towards the inward direction, following the given primary cosmic ray spectra. Before they are fed to the simulation code for the propagation in air, they are tested to determine whether they pass the rigidity cutoff or not. For a sampled cosmic ray, the "history" is examined by solving the equation of motion in the negative time direction. When the cosmic ray reaches the escape sphere without touching the injection sphere again in the inverse direction of time, the cosmic ray can pass through the magnetic barrier following its trajectory in the normal direction of time. The propagation of cosmic rays is simulated in the space between the surface of Earth and the simulation sphere.

In the 3-dimensional calculation of atmospheric neutrino flux, we need a very fast interaction code. On the other hand, the established interaction codes are generally slow in the actual computation. Therefore, we construct an inclusive interaction model from the output of the established interaction code, by the parameter fitting, and use it in the 3-dimensional calculation of atmospheric neutrino flux.

In Ref. [6], we used the inclusive interaction code constructed from the output of DPMJET-III [18], which is called as inclusive-DPMJET-III. After Ref. [17], we constructed the inclusive interaction code from the JAM interaction code, which is used in PHITS (Particle and HeavyIon Transport code System) [19], and used at the energies below $32 \mathrm{GeV}$.

\section{Hadronic interaction model and muon calibration}

The hadronic interaction model is one of the largest source of the uncertainty of the atmospheric neutrino flux. As the main source of the atmospheric neutrino is $\pi-\mu$ decay at low energies ( $\lesssim 100 \mathrm{GeV})$, we can use the observed muon spectra for the calibration of the hadronic interaction model, when we have well-determined primary cosmic ray spectra and accurately measured atmospheric muon spectra.
We consider the primary cosmic ray spectra in Fig. 1 are well determined below $100 \mathrm{GeV}$, since the observed data by AMS-01 [7] and BESS [8, 9] show a very good agreement. We used the atmospheric muon spectra observed mainly by BESS $[9,20,21]$. In this study we selected DPMJET-III [6], and modified it so that it reproduce the observed atmospheric muon spectra more accurately [22].

We note that the uncertainty of the cosmic ray spectra largely increases above $100 \mathrm{GeV}$. When we can use more accurate primary cosmic ray spectra above $100 \mathrm{GeV}$, we will repeat the study of the hadronic interaction model above $100 \mathrm{GeV}$ to improve the calculation of atmospheric neutrino flux. However, the energy of accurately measured atmospheric muon data is limited below a few TeV. The energy of the hadronic interaction studied with this method is also limited below a few $10 \mathrm{TeV}$,

\section{Calculated atmospheric neutrino flux}

In Fig. 4, we present the results for the atmospheric neutrino fluxes at $1 \mathrm{GeV}$ as a function of the zenith angle after averaging over all the azimuthal angles for INO [15] and South Pole [16]. The flux peak at the horizontal direction is due to the geometrical horizontal enhancement of the atmospheric neutrino flux. There are large up-down asymmetries in the atmospheric neutrino flux at both sites. The downward going neutrino flux is larger at the South pole, while upward going neutrino flux is larger at the INO site due to the different rigidity cutoff. These difference due to the rigidity cutoff decrease with the increase of neutrino energy, and almost disappear at $10 \mathrm{GeV}$.

Next we present the results for the atmospheric neutrino fluxes as a function of azimuthal angle $\phi$ for INO site (Fig. 5) and South Pole site (Fig.6). In these figures we show the variation of atmospheric neutrino flux as the function of the azimuthal angle averaging them over the five zenith angle ranges, $1>\cos \theta>0.6$, $0.6>\cos \theta>0.2,0.2>\cos \theta>-0.2,-0.2>\cos \theta>-$ 0.6 and $-0.6>\cos \theta>-1$. We find that the variation of the atmospheric neutrino flux has a complex structure at low (anti)neutrino energies, due to the rigidity cutoff and muon bending in the geomagnetic field. 

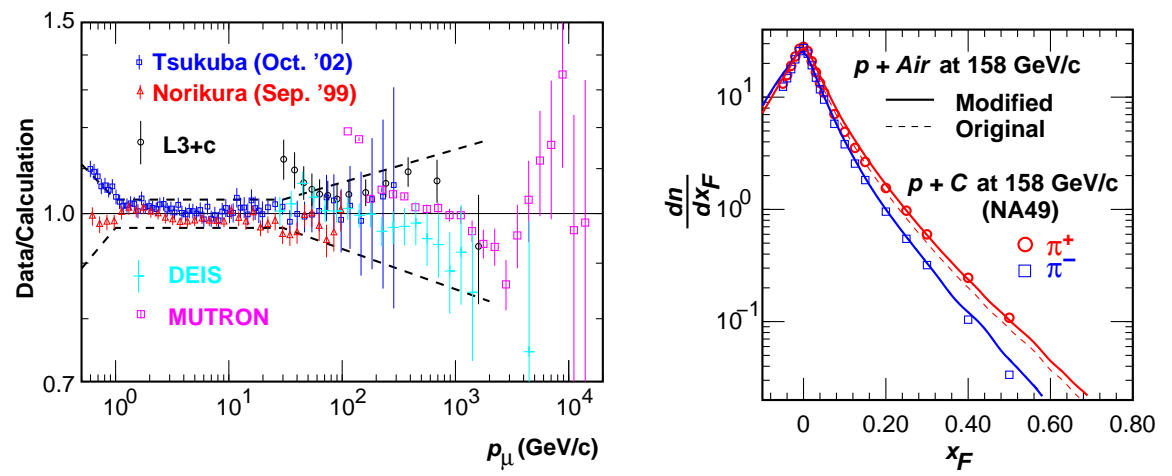

Figure 3. Left panel: comparison of observed muon spectra ad different observation points and calculation with modified hadronic interaction model. Norikura are from T. Sanuki et al. [21], Tsukuba from S. Haino et al. [9], L3+c from P. Achard et al. [24], DEIS from O.C. Allkofer et al. [25], and MUTRON from S. Matsuno et al. [26]. Right panel: comparison pion spectra of the accelerator data [23] and modified interaction model.
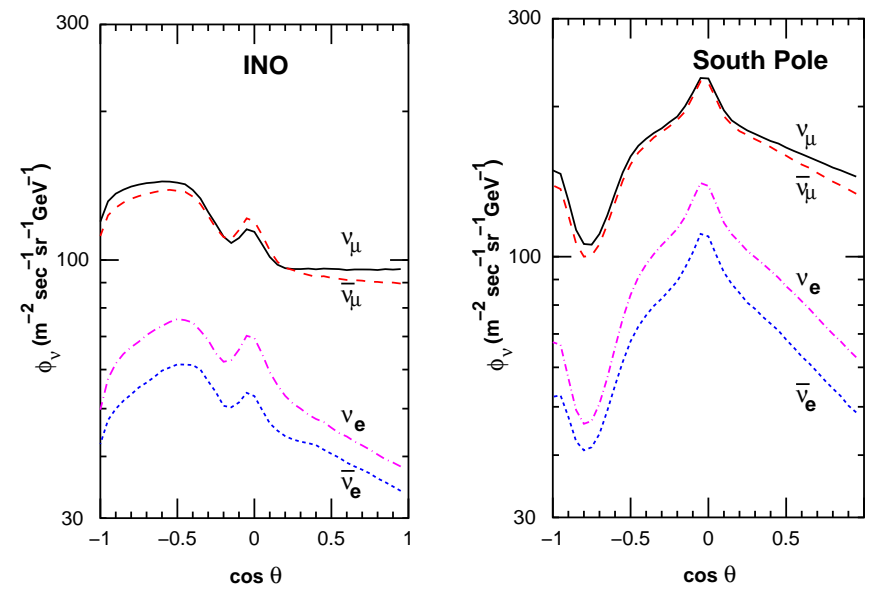

Figure 4. The zenith angle dependence of atmospheric neutrino flux at $\mathrm{E}=1 \mathrm{GeV}$, averaged over all azimuthal angles calculated for INO, South Pole and Pyhäsalmi sites. Here $\theta$ is the arrival direction of the neutrino, with $\cos \theta=1$ for vertically downward going neutrinos, and $\cos \theta=-1$ for vertically upward going neutrinos.

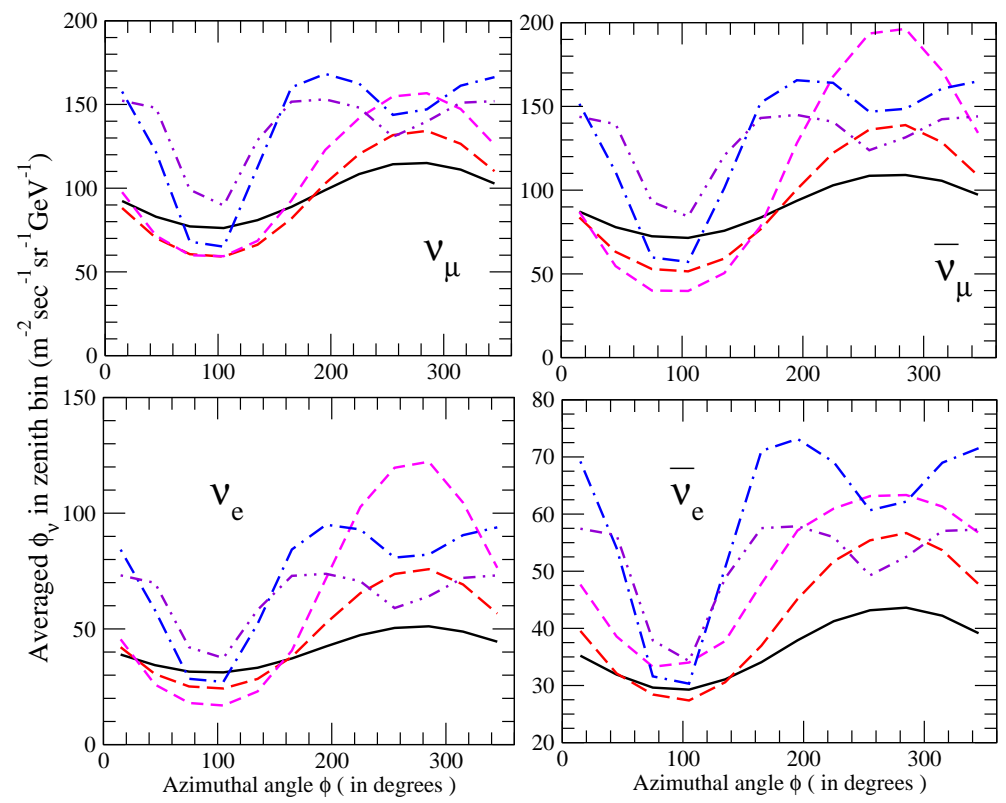

Figure 5. The azimuthal angle dependence of atmospheric neutrino flux, averaged over zenith angle bins of $1>\cos \theta>0.6$ (solid line), $0.6>\cos \theta>0.2$ (long dashed), $0.2>\cos \theta>-0.2$ (short dashed), $-0.2>\cos \theta>-0.6$ (dashed-dotted), and $-0.6>\cos \theta>-1$ (dashed double-dotted), calculated for the INO site at (anti)neutrino energy $\mathrm{E}=1 \mathrm{GeV}$. 


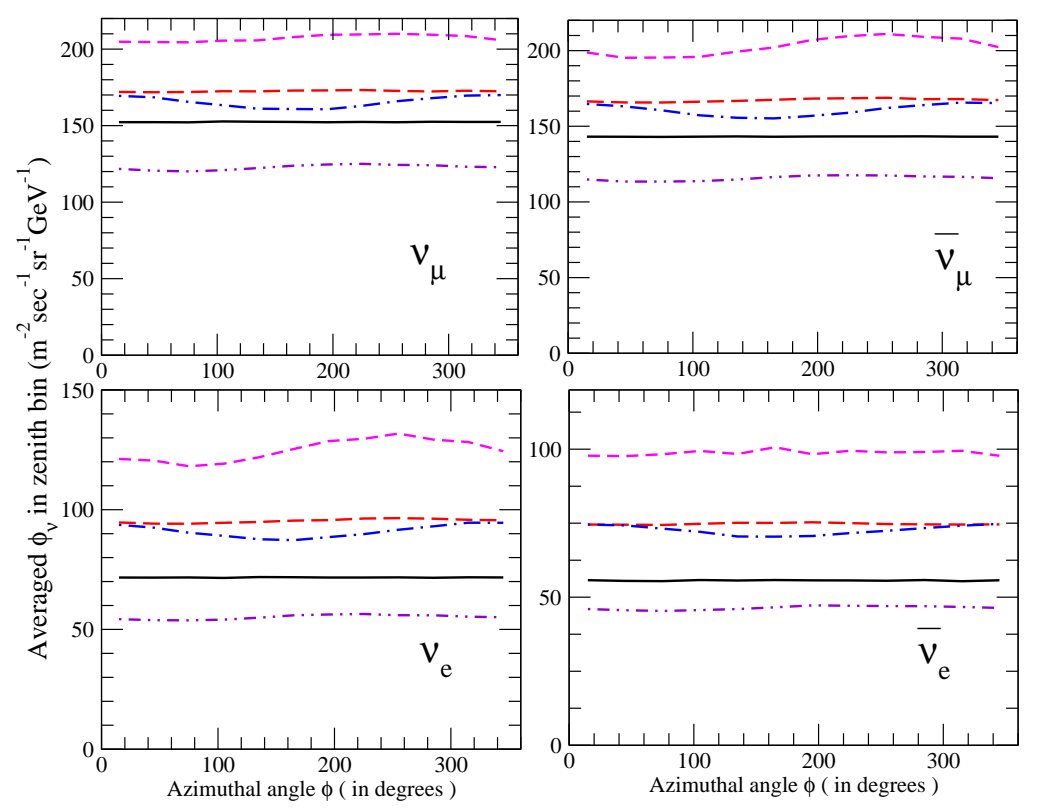

Figure 6. Same as Fig. 5 at (anti)neutrino energy $E=1 \mathrm{GeV}$ for the South Pole site.

At INO site the azimuth variation has the complex structure due to the high rigidity cutoff and the strong horizontal component of geomagnetic filed. This variation remains almost the same for the near horizontal direction even above $10 \mathrm{GeV}$. On the other hand, the azimuth variation at South Pole is very small due to the dipole nature of the geomagnetic field.

\section{Summary and discussions}

We find that the low energy atmospheric neutrino flux is quite different depending on the position in the geomagnetic field. Therefore, the full 3-dimensional scheme calculation is necessary for the theory prediction of atmospheric neutrino flux.

We use the hadronic interaction model after constructing the inclusive code, and it could be calibrated by the atmospheric muon spectra measured accurately. However, for the interaction above $10 \mathrm{TeV}$, the calibration with atmospheric muon is not effective, since there are no accurately measured muon spectra, and due to the uncertainty of primary cosmic ray spectra.

\section{Acknowledgments}

The author is grateful to the organizer of ISVHECRI for the hospitality. He also thanks to M.S. Athar, T. Kajita, K. Kasahara, S. Midorikawa, J. Nishimura, and A. Okada for the discussions.

\section{References}

[1] M. Honda, T. Kajita, K. Kahahara, S. Midorikawa, Phys. Rev. D54, 4985 (1995).
[2] V. Agrawal, T.K. Gaisser, P. Lipari, and T. Stanev, Phys. Rev. D53, 1314 (1996).

[3] G. Battistoni et al., Astropart. Phys. 12, 315 (2000).

[4] P. Lipari, Astropart. Phys. 14, 153 (2000).

[5] G.D. Barr et al., Phys. Rev. D70, 023006 (2004).

[6] M. Honda et al., Phys. Rev. D70, 043008 (2004).

[7] J. Alcaraz et al. (AMS-01), Phys. Lett. B 490, 27 (2000).

[8] T. Sanuki et al. (BESS), Astrophys. J. 545, 1135, (2000).

[9] S. Haino et al. (BESSTEV), Phys. Lett. B594352000.

[10] http://www.nrl.navy.mil/research/nrlreview/2003/atmospheric-science/picone

[11] http://www.ngdc.noaa.gov/IAGA/vmod/igrf.html.

[12] K. Asakimori et al., (JACEE) Astrophys. J.502 278 1998.

[13] A.V. Apanasenko, et al., (RUNJOB) Astropart. Phys. 16132001.

[14] M.S. Athar et al., hep-ph/1210.5154 .

[15] M. V. N. Murthy, A. I. P. Conf. Proc. 1405309 (2011).

[16] D. J. Koskinen, Mod. Phys. Lett. A 262899 (2011).

[17] M. Honda, et al., Phys. Rev. D831230012011.

[18] S. Roesler, R. Engel, and J. Ranft (2000), hep$\mathrm{ph} / 0012252$.

[19] K. Niita et al., Radiation Measurements 41 (2006) 1080 .

[20] K. Abe et al. (BESS), Phys. Lett. B 56482003.

[21] T. Sanuki et al. (BESS), Phys. Lett. B 5412342002.

[22] T. Sanuki, et al., Phys. Rev. D 750430052007.

[23] C. Alt et al. (NA49), Eur. Phys. J. C498972006

[24] P. Achard et al (L3+C). Phys. Lett. B598152004.

[25] O.C. Allkofer et al. (DEIS), Nucl. Phys. B25911985.

[26] S. Matsuno et al. (MUTRON), Phys. Rev. D2911984. 